\title{
Stress levels of higher education students during COVID-19 and their correlation with diet quality and lifestyle behaviours
}

\author{
S. Doak ${ }^{1}$, J. Kearney ${ }^{2}$, J.M. McCormack ${ }^{1}$ and L. Keaver ${ }^{1}$ \\ ${ }^{1}$ Department of Health and Nutritional Science, Institute of Technology Sligo, Sligo, Ireland and \\ ${ }^{2}$ School of Biological and Health Sciences, Technological University Dublin, Dublin, Ireland
}

Higher education students tend to experience elevated levels of stress ${ }^{(1)}$, which are strongly associated with poor academic performance $^{(2)}$. Academic impairment increases the likelihood of college dropout, therefore, a prominent issue for higher education institutes to consider ${ }^{(3)}$. In other countries, having healthier patterns of diet and lifestyle behaviours are associated with lower levels of stress in students $^{(4)}$. The aim of this study was to assess the current perceived stress levels and their relationship with diet and lifestyle behaviours in a sample of Irish higher education students.

Full-time students, aged 18 and over, were eligible to participate in this cross-sectional web-based survey, hosted by Qualtrics ${ }^{\circledR}$ XM. Recruitment was conducted in the 2020-2021 academic year, during varying stages of the COVID-19 pandemic in three Irish institutes. Self-reported information was collected in relation to demographics, BMI, smoking and the impact of COVID-19. Stress levels of students were measured using the validated Perceived Stress Scale-10 - (PSS-10), which was completed along with validated measures assessing dietary quality (short-form food frequency questionnaire), alcohol use (Alcohol Use Disorders Identification Test Alcohol Consumption - AUDIT- C), sleep quality (Pittsburgh Sleep Quality Index - PSQI), and physical activity (International Physical Activity Questionnaire Short Form - IPAQ-SF). Statistical analysis was performed using Pearson's product-moment correlation and a one-way ANOVA test. Significance was set at $\mathrm{p}<0.05$.

A total of 682 , mainly female $(n=455,66.8 \%)$ students completed the survey cohort, with a mean age of $24.97 \pm 8.90$ years. Mean PSS-10 scores were $16.78 \pm 5.37$ with over two-thirds $(n=500,73.3 \%)$ of students deemed moderately or highly stressed. Most students $(n=411,62.1 \%)$ expressed that they were more stressed than usual due to the COVID-19 pandemic with few $(n=57,8.6 \%)$ reporting feeling less stressed. There was a statistically significant, small correlation found between PSS-10 scores and PSQI scores (sleep quality) $(r(672)=0.195, p<0.001)$, and a negative correlation between PSS-10 scores and diet quality $(r(682)=-0.104, p$ $=0.006)$, fruit and vegetable $(\mathrm{F} \& \mathrm{~V})$ consumption $(r(676)=-0.158, p<0.001)$, and physical activity scores $(r(665)=-0.160, p$ $<0.001)$. There was no statistically significant correlation between PSS-10 scores and smoking status $(p=0.924)$, AUDIT-C scores $(p=0.221)$ or BMI $(p=0.569)$.

There is evidence of a correlation between having higher levels of stress and a lower quality diet, lower F\&V consumption, being less physically active, and having poor sleep quality. BMI, alcohol consumption, and being a smoker were not associated with the stress levels of students. Additional research and clarity of the relationship between stress levels and the diet and lifestyle behaviours of students are warranted in this population group to inform the development of initiatives and programmes to reduce the stress levels of students and potentially decrease the likelihood of college dropout ${ }^{(4)}$.

\section{Acknowledgments}

The authors wish to thank Jason Quinn, Keelan Kennoy and Ryan O'Reilly from ITS Students' Union, Yvonne Roache from ITS, Colin Kearney and Victor O'Loughlin from GMIT Students' Union and Mary Hernandez from LYIT Students' Union for helping with survey promotion. In addition, the authors thank the students who participated in this study as volunteers, making the study possible.

\section{References}

1. Du C, Zan MCH, Cho MJ et al. (2021) Nutrients 13(2), 442.

2. Reuter PR, Forster BL \& Brister SR (2020) J Am Coll Health, 1-7.

3. Turner K \& McCarthy VL (2017) Nurse Educ Pract 22, 21-29.

4. Hanawi SA, Saat NZM, Zulkafly M et al. (2020) Int J Pharm Res Allied Sci 9(2), 1-7. 